\title{
Numerical Study between Conservative and Non-conservative form of a Traffic Flow Model
}

\author{
Md. Shajib Ali ${ }^{1 *}$, M.M.Rahman ${ }^{2}$, and Most R.Khatun ${ }^{3}$
}

\author{
${ }^{1}$ Dept. of Mathematics, Islamic University, Kushtia, Bangladesh \\ ${ }^{2}$ Dept. of Mathematics, Patuakhali Science and Technology University, Patuakhali, Bangladesh \\ ${ }^{3}$ Dept. of Mathematics, Bangabandhu Sheikh Mujibur Rahman Science and Technology University, Gopalganj, Bangladesh
}

DOI: $10.36347 /$ sipms.2022.v09i01.003

| Received: 29.11.2021 | Accepted: 04.01.2022 | Published: 30.01.2022

*Corresponding author: Md. Shajib Ali

Dept. of Mathematics, Islamic University, Kushtia, Bangladesh

Abstract

A conservative and non-conservative form of a macroscopic traffic flow model which are non-linear first order partial differential equation appended with initial and boundary condition that formulates an initial boundary value problem (IBVP). In analytical methods traffic flow model is too complex to be solved and due to the complexity of findings the analytical solution we investigate numerical solution by finite difference method. For numerical solution we present finite difference scheme namely as explicit upwind difference scheme for conservative and non-conservative form of a single lane traffic flow model and performs a comparative study for these numerical scheme to understand the computational complexity and efficiency of the schemes.

Keywords: Traffic flow model, Finite difference scheme and Numerical simulation.

Copyright $(\mathcal{2 0 2 2}$ The Author(s): This is an open-access article distributed under the terms of the Creative Commons Attribution 4.0 International License (CC BY-NC 4.0) which permits unrestricted use, distribution, and reproduction in any medium for non-commercial use provided the original author and source are credited.

\section{INTRODUCTION}

Every day the demand for travel increases and consequently roads are becoming more congested. Congestion results in increased travel times and exhaust fume emissions. Now a days, in our country, the fast growing number of vehicles on urban streets and roadways together with related economic and social implications, such as, prevention of car crashes, pollution and energy control, has motivated to go into this research activity in the field of traffic flow modeling. Traffic flow and congestion is related to our transportation. Information technology is making available new methods for the measuring, control and optimization of motorway traffic. Traffic phenomena are complex and nonlinear, depending on the interactions of a large number of vehicles. Many research groups are involved in dealing with the problem with different kinds of traffic models like the microscopic car following model, the macroscopic fluid dynamic model and the mesoscopic (Kinetic) model. All models describe various situations with different assumptions and simplifications.

A macroscopic theory of traffic can be developed with the help of hydrodynamic theory of fluids by considering traffic as an affectivity one dimensional compressible fluid. The macroscopic traffic flow theory was introduced in the fluid-dynamic model of Michael James Lighthill, Gerald Beresford Whitham and Paul Richards (or the LWR model) for describing traffic flows and car following experiments $([1,4,5])$. This well-known paper of Lighthill and Whithham published in 1955 and introduce a description based on the equation of continuity, together with the assumption that flow (or velocity) depends on the density only, i.e. there is no relaxation time, velocity adapts instantaneously to the surrounding density.

As presented, we study finite difference method for first order non-linear PDE ([6, 8, 9, 10]) and based on these, we develop finite difference schemes for conservative and non-conservative form of traffic flow model as an (IBVP) which has been presented in numerical simulation. We develop computer programming code for the implementation of the numerical schemes and perform numerical experiments in order to compare the efficiency and some qualitative behavior of conservative and non-conservative form of traffic flow for various traffic parameters of the numerical simulation. 


\section{Mathematical equation of conservative and non-} conservative form of a traffic flow model

The well-known LWR traffic flow model ([4, 5]) based on the principle of mass conservation. The among relationship velocity, density and flux, the flux $q=\rho v$ yields the equation of continuity.

$\frac{\partial \rho}{\partial t}+\frac{\partial q}{\partial x}=0$

The interpretation and construction of the velocity-density relationship plays a vital role in the macroscopic traffic flow model. We consider velocity $v=v(\rho)$ as a function of density and therefore, we have the flux $q=q(\rho)=\rho v(\rho)$. The first steadystate velocity-density relation is introduced by Greenshields, who proposed a linear relationship between velocity-density that is as and also a non-linear velocity-density (non-linear function) which is of the form

$$
v(\rho)=v_{\max }\left(1-\left(\frac{\rho}{\rho_{\max }}\right)^{2}\right)
$$

Where, $v_{\max }$ denotes maximum velocity (free flow speed) and $\rho_{\max }$ denotes maximum density (jam density). We use a linear velocity-density $v(\rho)=v_{\max }\left(1-\frac{\rho}{\rho_{\max }}\right)$ and the flux $q$ takes the form $\quad q=q(\rho)=\rho v(\rho)$ $=\rho \cdot v_{\max }\left(1-\frac{\rho}{\rho_{\max }}\right)=v_{\max }\left(\rho-\frac{\rho^{2}}{\rho_{\max }}\right)$

$v(\rho)=v_{\max }\left(1-\frac{\rho}{\rho_{\max }}\right)$

Equation (1) leads to formulate $\frac{\partial \rho}{\partial t}+\frac{\partial q(\rho)}{\partial x}=0$ where $q(\rho)=v_{\max }\left(\rho-\frac{\rho^{2}}{\rho_{\max }}\right)$ and $\frac{\partial \rho}{\partial t}+\frac{\partial q(\rho)}{\partial x}=0$ that is $\frac{\partial \rho}{\partial t}+q^{\prime}(\rho) \frac{\partial \rho}{\partial x}=0$ where $q^{\prime}(\rho)=v_{\max }\left(1-\frac{2 \rho}{\rho_{\max }}\right)$.

Therefore the mathematical equations of traffic flow model with the initial condition reads as initial value problem (IVP) ([3], [4], and [5]) are

\section{Conservative form}

$$
\begin{aligned}
& \frac{\partial \rho}{\partial t}+\frac{\partial q(\rho)}{\partial x}=0 ; q(\rho)=v_{\max }\left(\rho-\frac{\rho^{2}}{\rho_{\max }}\right) \\
& \text { with } \rho\left(t_{0}, x\right)=\rho_{0}(x)
\end{aligned}
$$

\section{Non-conservative form}

$$
\begin{aligned}
& \frac{\partial \rho}{\partial t}+q^{\prime}(\rho) \frac{\partial \rho}{\partial x}=0 ; q^{\prime}(\rho)=v_{\max }\left(1-\frac{2 \rho}{\rho_{\max }}\right) \\
& \text { with } \rho\left(t_{0}, x\right)=\rho_{0}(x)
\end{aligned}
$$

\section{Analytic solution of conservative and non-} conservative form of traffic flow model

The non-linear PDE of IVP (2) and (3) can be solved [9] by the method of characteristics. The exact solution is given by [2].

$$
\rho(t, x)=\rho_{0}\left(x-v_{\max }\left(1-\frac{2 \rho}{\rho_{\max }}\right) t\right)
$$

Which is very complicated to evaluate at each $\rho(t, x)$. Therefore, there is a demand of some efficient numerical methods for solving the IVP (2) and (3).

\section{Numerical methods of conservative and non- conservative form of traffic flow Model}

We consider our non-linear first order partial differential equation of traffic flow model as an initial boundary value problem (IBVP):

\section{Conservative form}

$$
\begin{aligned}
& \frac{\partial \rho}{\partial t}+\frac{\partial q(\rho)}{\partial x}=0 \text {; where } q(\rho)=v_{\max }\left(\rho-\frac{\rho^{2}}{\rho_{\max }}\right) \\
& \text { with I.C. } \rho\left(t_{0}, x\right)=\rho_{0}(x) \text { and B.C. } \rho(t, a)=\rho_{a}(t) .
\end{aligned}
$$

\section{Non-conservative form}

$$
\begin{aligned}
& \frac{\partial \rho}{\partial t}+q^{\prime}(\rho) \frac{\partial \rho}{\partial x}=0 \text {; where } q^{\prime}(\rho)=v_{\max }\left(1-\frac{2 \rho}{\rho_{\max }}\right) \\
& \text { with I.C. } \rho\left(t_{0}, x\right)=\rho_{0}(x) \text { and B.C. } \rho(t, a)=\rho_{a}(t) .
\end{aligned}
$$

To establish this scheme, we discretize the time derivative $\frac{\partial \rho}{\partial t}$ and space derivative $\frac{\partial q}{\partial x}$ in the IBVP (5) and also discretize the time 
Derivative $\frac{\partial \rho}{\partial t}$ and space derivative $\frac{\partial \rho}{\partial x}$ in the IBVP (6) at any discrete point $\left(t_{n}, x_{i}\right)$ for $i=1, \ldots \ldots, M$ and $j=0, \ldots \ldots ., N-1$.

We assume the uniform grid spacing $t^{n+1}=t^{n}+k$ and $x_{i+1}=x_{i}+h$. The discretization of $\frac{\partial \rho}{\partial t}$ is obtained by first order forward difference in time and the

Putting equation (7) and (8) in equation (5) and writing $\rho_{i}^{n}$ for $\rho\left(t^{n}, x_{i}\right)$, the discrete version of the

$$
\begin{gathered}
\frac{\rho_{i}^{n+1}-\rho_{i}^{n}}{\Delta t}+\frac{q_{i}^{n}-q_{i-1}^{n}}{\Delta x}=0 \\
\Rightarrow \rho_{i}^{n+1}=\rho_{i}^{n}-\frac{\Delta t}{\Delta x}\left(q_{i}^{n}-q_{i-1}^{n}\right)
\end{gathered}
$$$$
\text { where } q\left(\rho_{i}^{n}\right)=v_{\max }\left(\rho_{i}^{n}-\frac{\left(\rho_{i}^{n}\right)^{2}}{\rho_{\max }}\right) \text { and } q\left(\rho_{i-1}^{n}\right)=v_{\max }\left(\rho_{i-1}^{n}-\frac{\left(\rho_{i-1}^{n}\right)^{2}}{\rho_{\max }}\right) \text {. }
$$
upwind difference scheme of the conservative form

And $\frac{\partial \rho\left(t_{n}, x_{i}\right)}{\partial x} \approx \frac{\rho_{i}^{n}-\rho_{i-1}^{n}}{\Delta x}$

non-linear PDE formulates the first order explicit

Again, equation (7) and (9) in equation (6) and writing $\rho_{i}^{n}$ for $\rho\left(t^{n}, x_{i}\right)$, the discrete version of the

$$
\begin{aligned}
& \frac{\rho_{i}^{n+1}-\rho_{i}^{n}}{\Delta t}+q^{\prime}\left(\rho_{i}^{n}\right) \frac{\rho_{i}^{n}-\rho_{i-1}^{n}}{\Delta x}=0 \\
& \Rightarrow \rho_{i}^{n+1}=\rho_{i}^{n}-q^{\prime}\left(\rho_{i}^{n}\right) \frac{\Delta t}{\Delta x}\left(\rho_{i}^{n}-\rho_{i-1}^{n}\right) \\
& \text { where } q^{\prime}\left(\rho_{i}^{n}\right)=v_{\max }\left(1-\frac{2 \rho_{i}^{n}}{\rho_{\max }}\right) .
\end{aligned}
$$

The equations (10) and (11) are the explicit finite difference schemes for IBVP (4) and (5). In the finite difference scheme, the initial and boundary data $\rho_{i}^{0}$ and $\rho_{a}^{n}$ for all $i=1,2, \ldots \ldots, M$ and $j=0,1, \ldots \ldots ., N-1$ are the discrete versions of the given initial and boundary values $\rho_{0}(x)$ and $\rho_{a}(t)$ respectively. non-linear PDE formulates the first order explicit upwind difference scheme of the non-conservative form

In explicit upwind difference scheme for nonlinear PDE of traffic flow maximum velocity is unknown but fortunately it is known in our specific model by the velocity-density relationship $q\left(\rho_{i}^{n}\right)=v_{\max }\left(\rho_{i}^{n}-\frac{\left(\rho_{i}^{n}\right)^{2}}{\rho_{\max }}\right)$ i.e.
$q^{\prime}\left(\rho_{i}^{n}\right)=v_{\max }\left(1-\frac{2 \rho_{i}^{n}}{\rho_{\max }}\right) \geq 0$

\subsection{Well-posed-ness and stability condition}

$$
\Rightarrow \rho_{\max } \geq 2 \rho_{i}^{n} \text { i.e. } q^{\prime}\left(\rho_{i}^{n}\right) \leq v_{\max }
$$

Which is the condition for well-posed-ness

The explicit finite difference scheme (11) takes the form 


$$
\begin{aligned}
& \rho_{i}^{n+1}=\rho_{i}^{n}-q^{\prime}\left(\rho_{i}^{n}\right) \frac{\Delta t}{\Delta x}\left(\rho_{i}^{n}-\rho_{i-1}^{n}\right) \\
& \Rightarrow \rho_{i}^{n+1}=(1-\lambda) \rho_{i}^{n}+\lambda \rho_{i-1}^{n}
\end{aligned}
$$

where $\lambda:=q^{\prime}\left(\rho_{i}^{n}\right) \frac{\Delta t}{\Delta x}$. The equation (13) implies that if $\lambda \leq 1$, the new solution is a convex combination of the two previous solutions. That is the solution at new time-step $(n+1)$ at a spatial node is an average of the solutions at the previous time-step at the spatial nodes $i$ and $i-1$. This means that the extreme value of the new solution is the average of the extreme values of the previous two solutions at the two consecutive nodes. Therefore, the new solution continuously depends on the initial value $\rho_{i}^{o}, i=1,2,3, \ldots \ldots ., M$ and the explicit finite difference scheme is stable for $\lambda:=q^{\prime}\left(\rho_{i}^{n}\right) \frac{\Delta t}{\Delta x} \leq 1$ and then by condition in equation (12) implies that

$\lambda:=\frac{v_{\max } \Delta t}{\Delta x} \leq 1$

This is the stability condition. Thus whenever one employs the stability condition $\lambda:=v_{\max } \frac{\Delta t}{\Delta x} \leq 1$, the well-posed-ness condition equation (12) can be guaranteed immediately by choosing $\rho_{\max }=k \max _{i} \rho_{\mathrm{o}}\left(x_{i}\right), k \geq 2$.

\section{Numerical experiments and results discussion}

In this section, we present numerical results for some specific cases of traffic flow focusing on the traffic flow parameters of a single lane highway. We choose maximum velocity $v_{\max }=60 \mathrm{~km} /$ hour. For satisfying the CFL condition we pick the unit of velocity as $\mathrm{km} / \mathrm{sec}$. We consider $\rho_{\max }=550 / \mathrm{km}$, and perform the numerical experiment for 6 minutes in 3600 time steps with $\Delta t=0.1$ second for a single lane highway of $10 \mathrm{~km}$ in 401 spatial grid points with step size $\Delta \mathrm{x}=100$ meters. We consider the initial density of traffic flow model for single lane is $\rho(0, x)$ and take $\alpha=10$ as a constant and also the constant one sided boundary value for EUDS is $\rho(t, 0)=21 / 0.1 \mathrm{~km}$ to perform numerical computation in the spatial domain $[0,10]$ in $\mathrm{km}$. We simulate the traffic flow for six minutes. Using initial and boundary condition on EUDS scheme, we can forecast the traffic flow model. In figure-1 presents initial density profile conservative and non-conservative form. Figure-2 shows after six minutes position of traffic density profile.
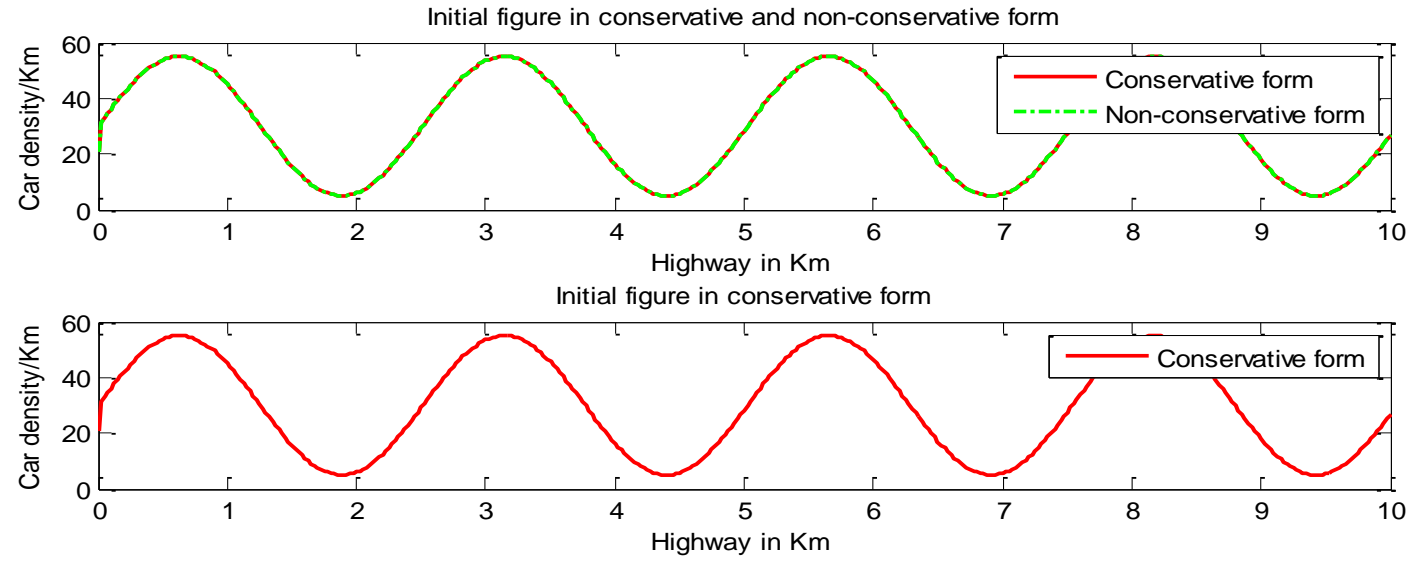

Initial figure in non-conservative form

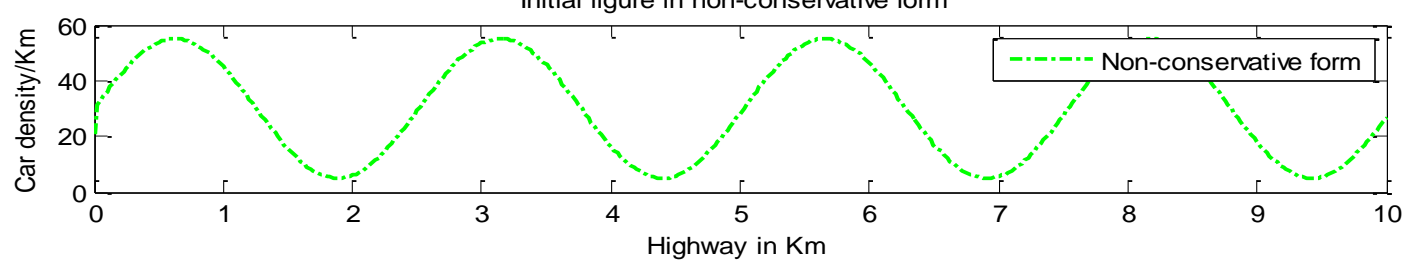

Fig-1: Initial density conservative and non-conservative form in a $10 \mathrm{~km}$ highway 

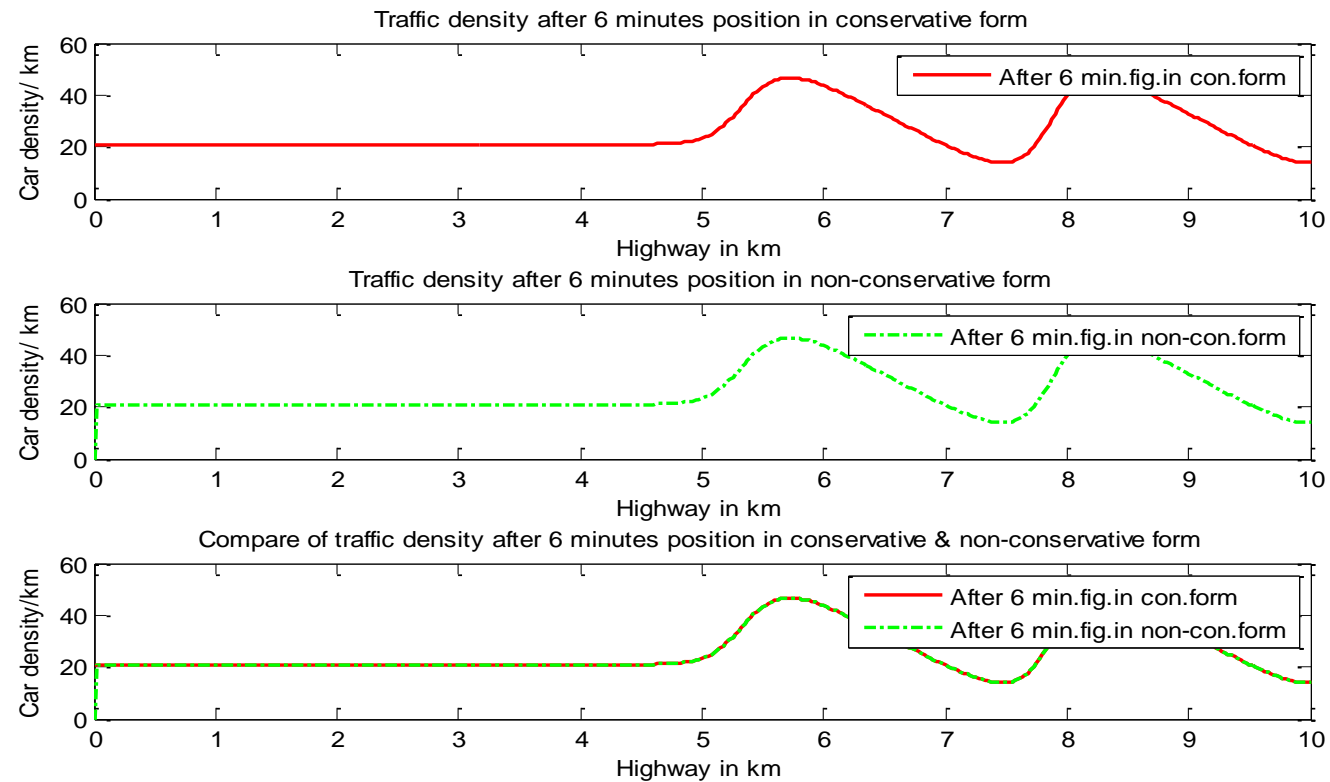

Fig-2: Traffic density profile after 6 minutes in conservative and non-conservative form

In figure-3(a) the curve marked by solid red line represents the density of car at 2, 4, 6 minutes of conservative form and dashes green line represents the density profile of traffic flow in non-conservative form at 2, 4, 6 minutes respectively. Figure-3(b)(i) \& 3(b)(ii) represents the respective computed velocity profile according to the certain points of a single lane highway. The velocity is computed by the following relation $v(\rho)=v_{\max }\left(1-\frac{\rho}{\rho_{\max }}\right)($ linear $\quad$ case $) \quad$ and $v(\rho)=v_{\max }\left(1-\left(\frac{\rho}{\rho_{\max }}\right)^{2}\right)$ (non-linear case). Now we know the traffic density and speed for certain points. So we calculate the flux with the aid of the relation $q=\rho v$. Figure 3(c)(i) \& 3(c)(ii) represents the computed flux (linear and non-linear case) with respected to the distance in conservative and nonconservative form.
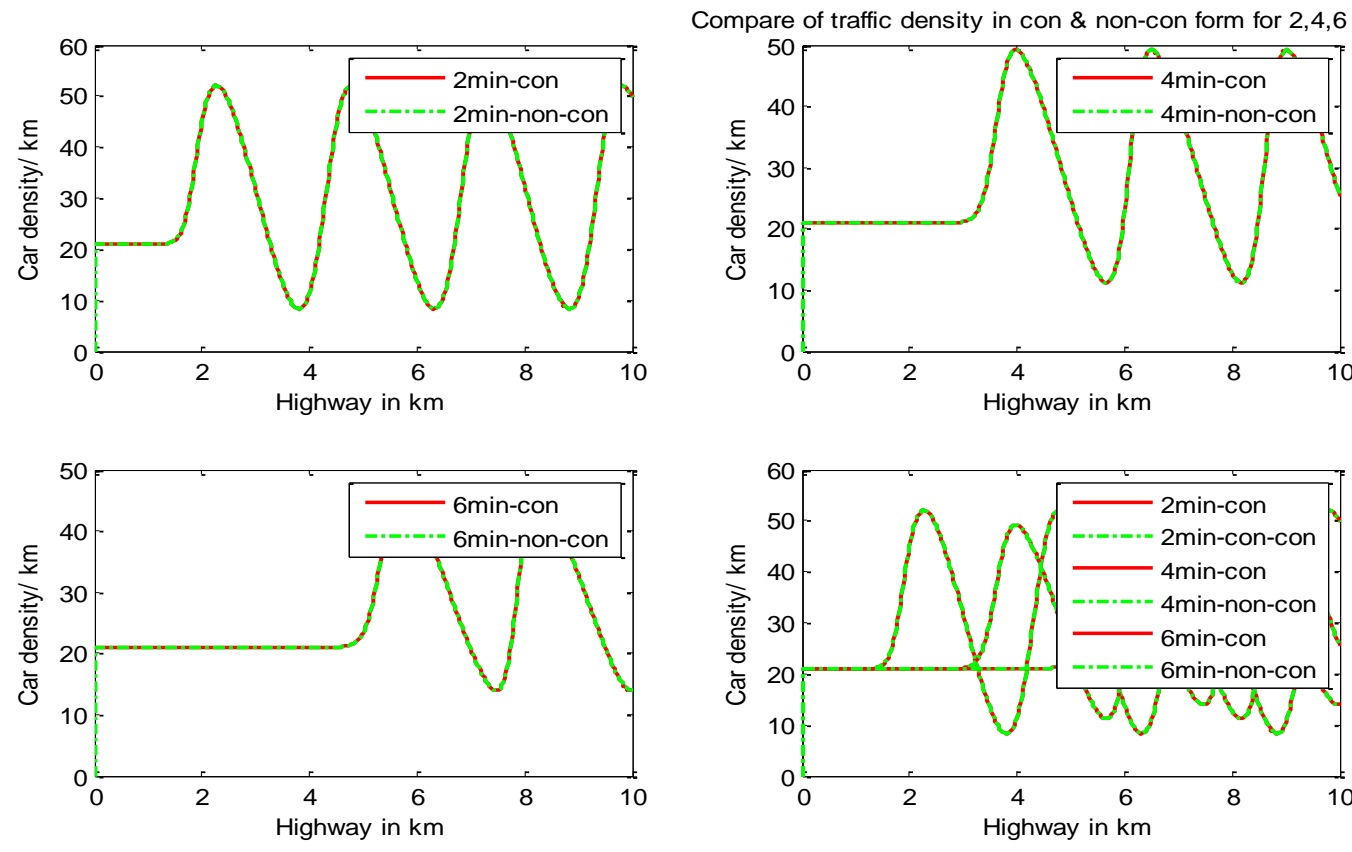

Fig-3(a): Density profile conservative and non-conservative form of 2, 4, 6 minutes in a $10 \mathrm{~km}$ highway 

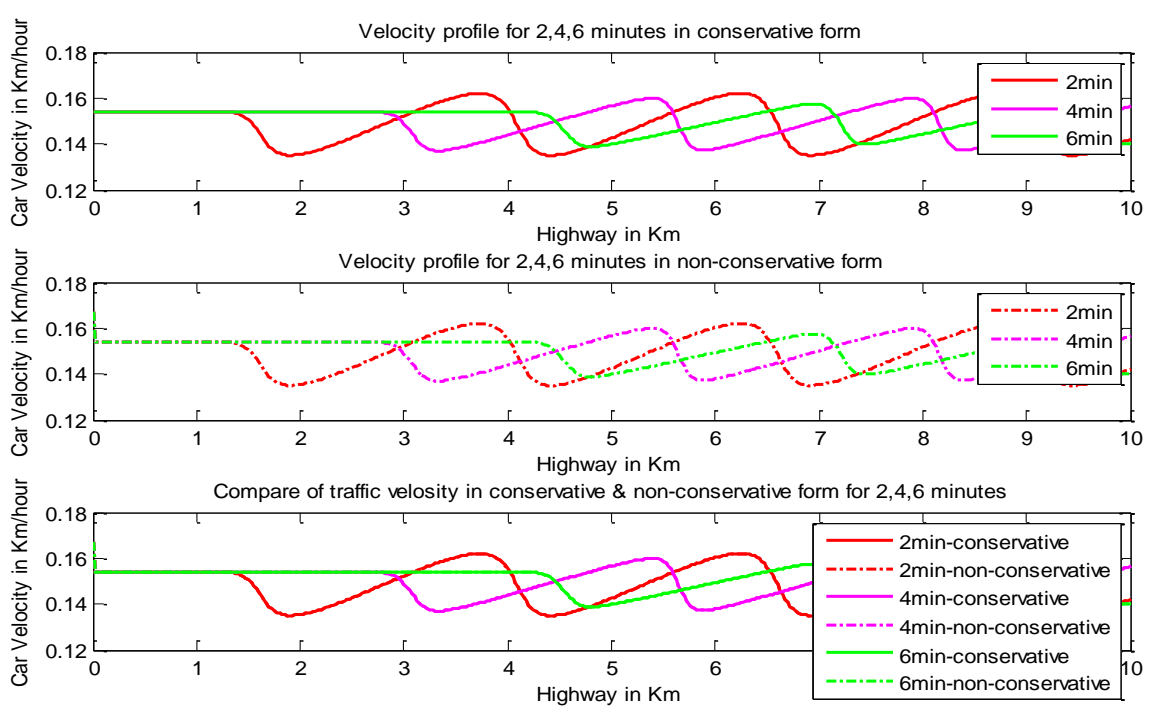

Fig-3(b)(i): Velocity profile conservative and non-conservative form of 2, 4, 6 minutes (linear) in a $10 \mathrm{~km}$ highway
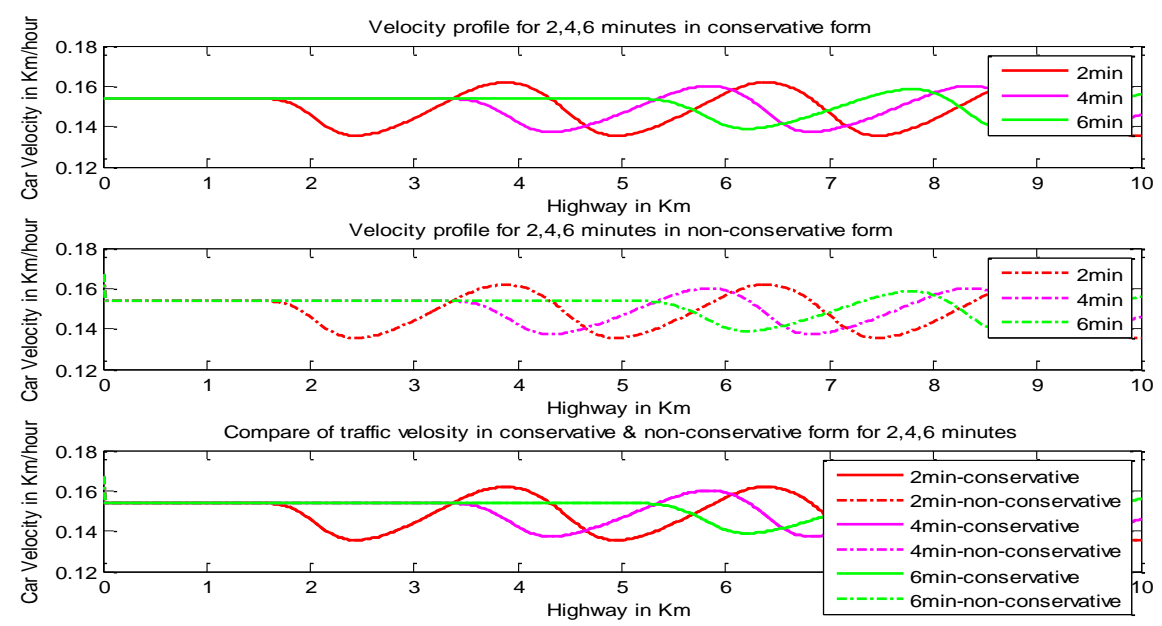

Fig-3(b)(ii): Velocity profile conservative and non-conservative form of $2,4,6$ minutes (non-linear) in a $10 \mathrm{~km}$ highway

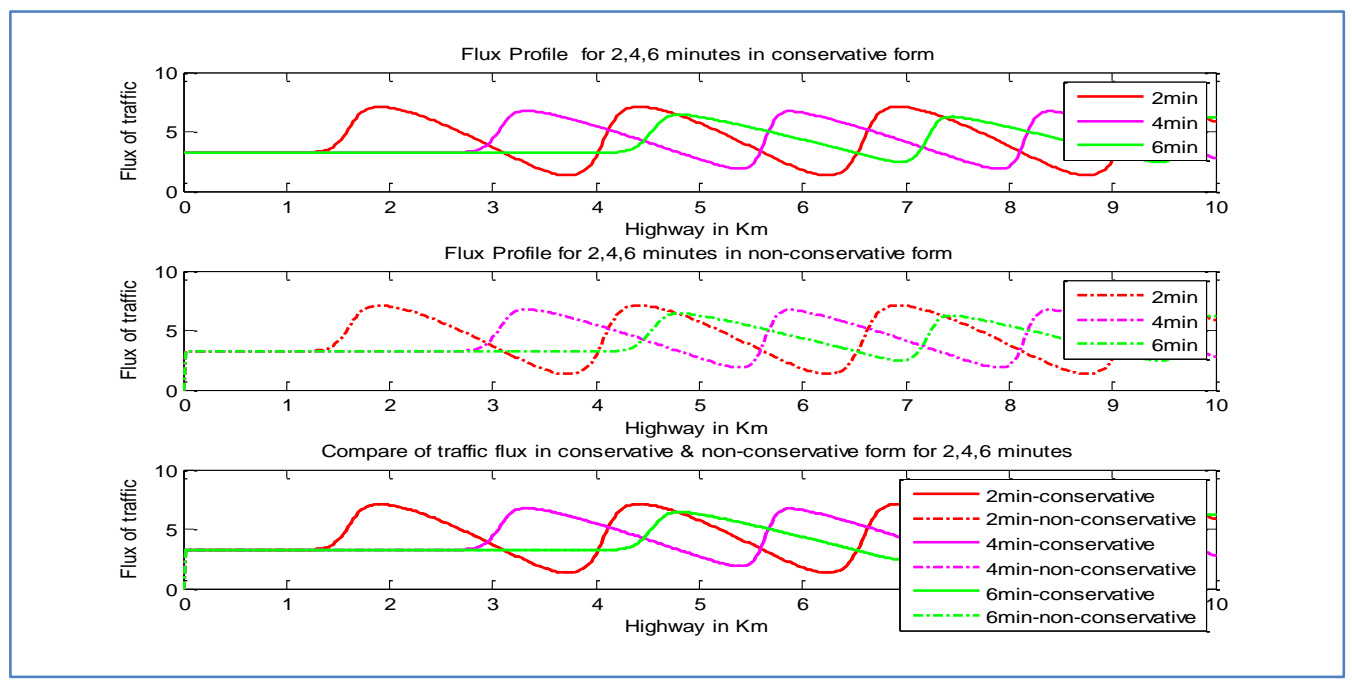

Fig-3(c)(i): Flux profile conservative and non-conservative form of 2, 4, 6 minutes (linear) in a $10 \mathrm{~km}$ highway 


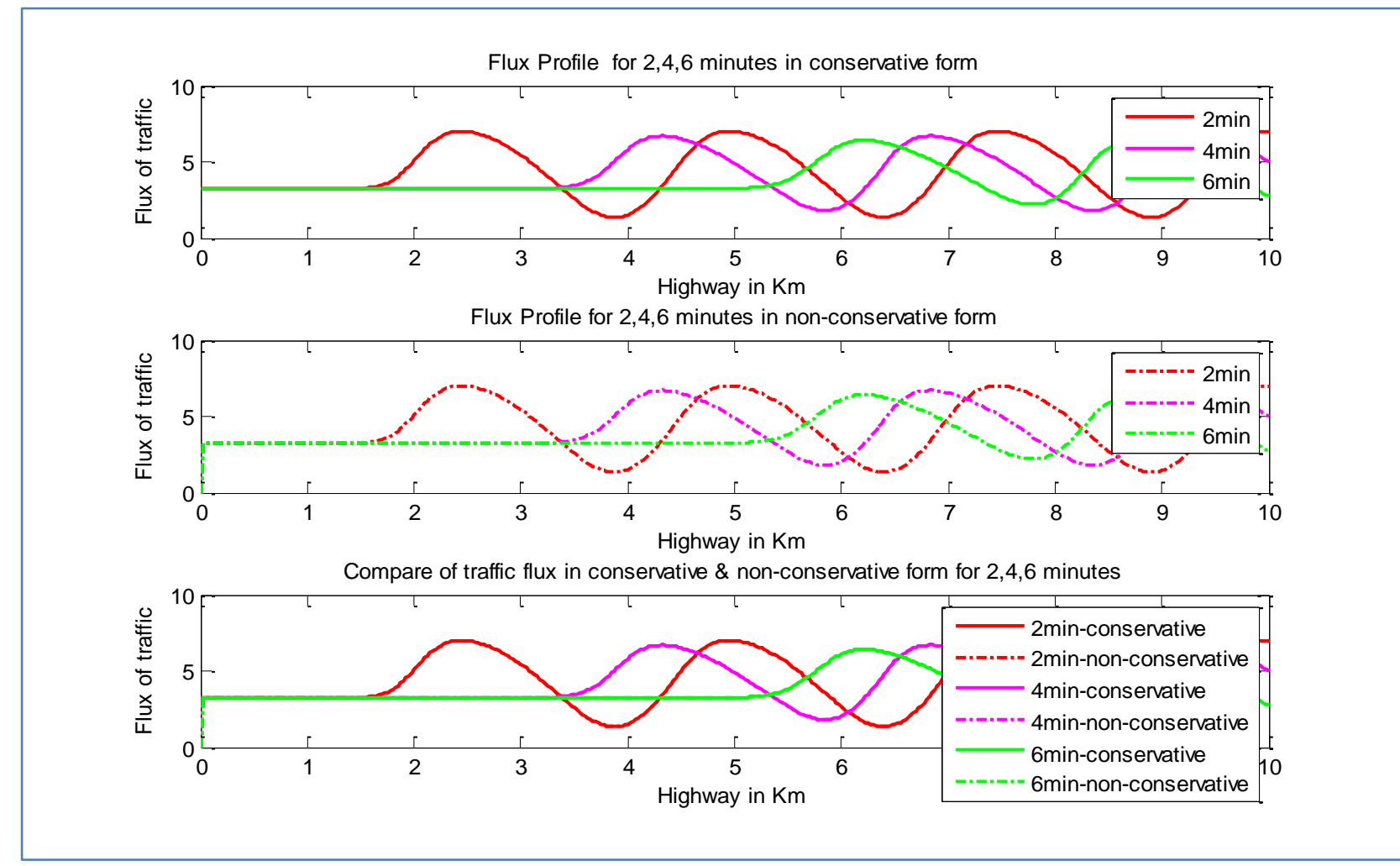

Fig-3(c)(ii): Flux profile conservative and non-conservative form of 2, 4, 6 minutes (non-linear) in a $10 \mathrm{~km}$ highway

In figure-4(i) and 4(ii) plot the computed velocity profile with respect to the computed density profile by the formula $v(\rho)=v_{\max }\left(1-\frac{\rho}{\rho_{\max }}\right)$ and $v(\rho)=v_{\max }\left(1-\left(\frac{\rho}{\rho_{\max }}\right)^{2}\right)$ of a traffic flow in conservative and non-conservative form. The figure shows that the velocity and density relationship is linear which agrees accurately with our assumptions.

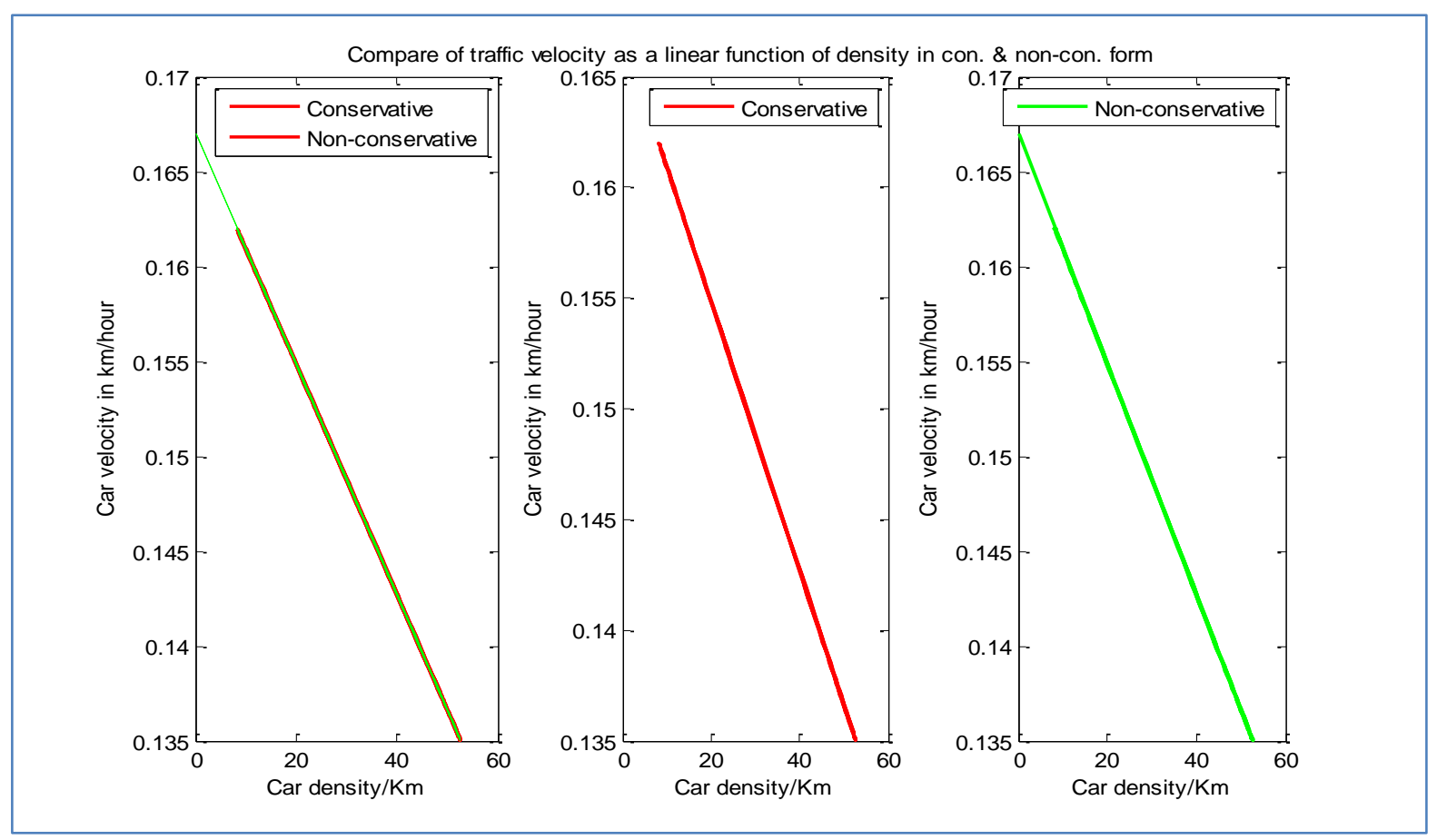

Fig-4(i): Traffic velocity as a function of density (linear case) conservative and non-conservative form 

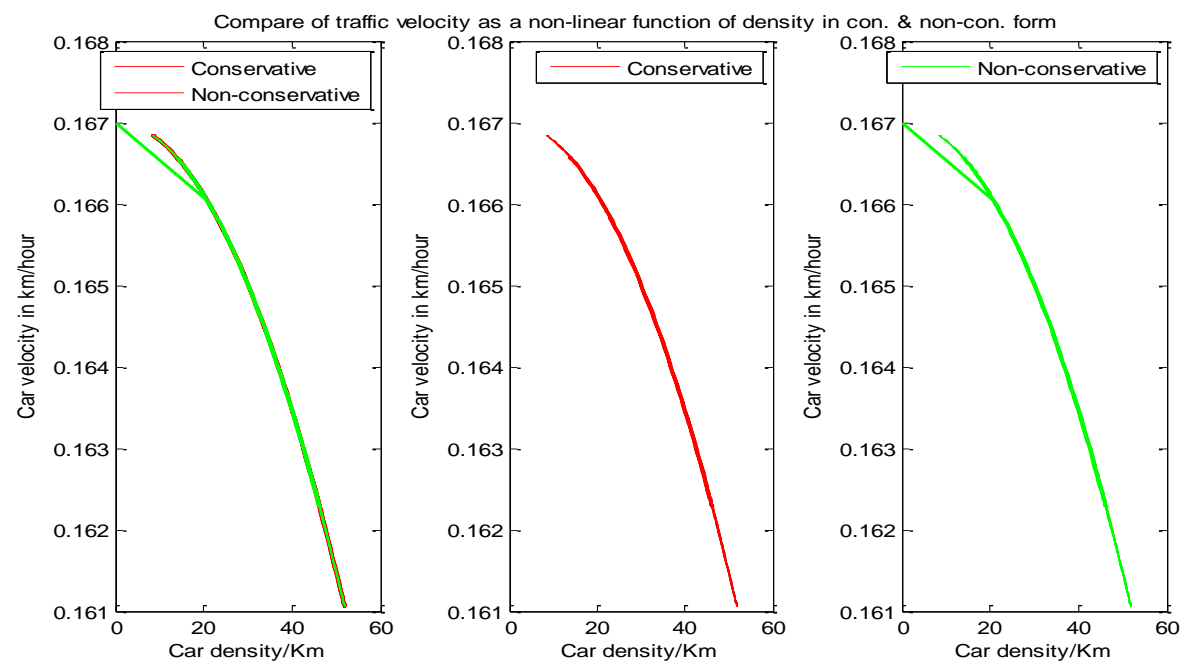

Fig-4(ii): Traffic velocity as a function of density (non-linear case) conservative and non-conservative form

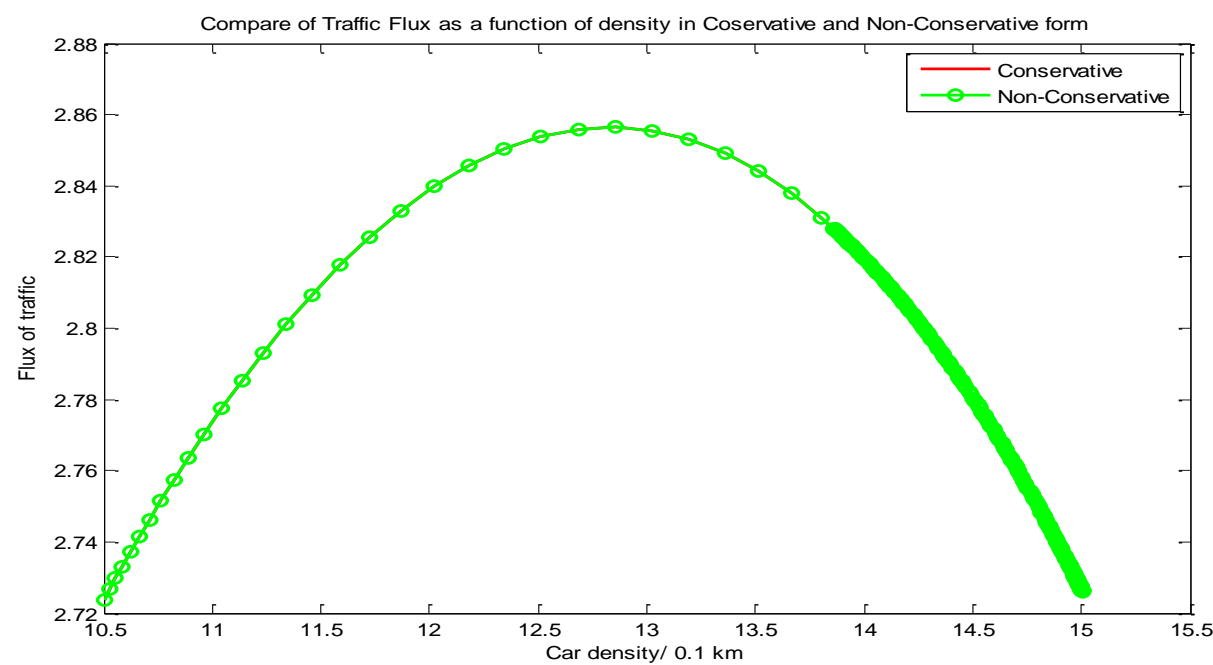

Fig-5(i): Traffic Flux as a function of density (linear case) conservative and non-conservative form

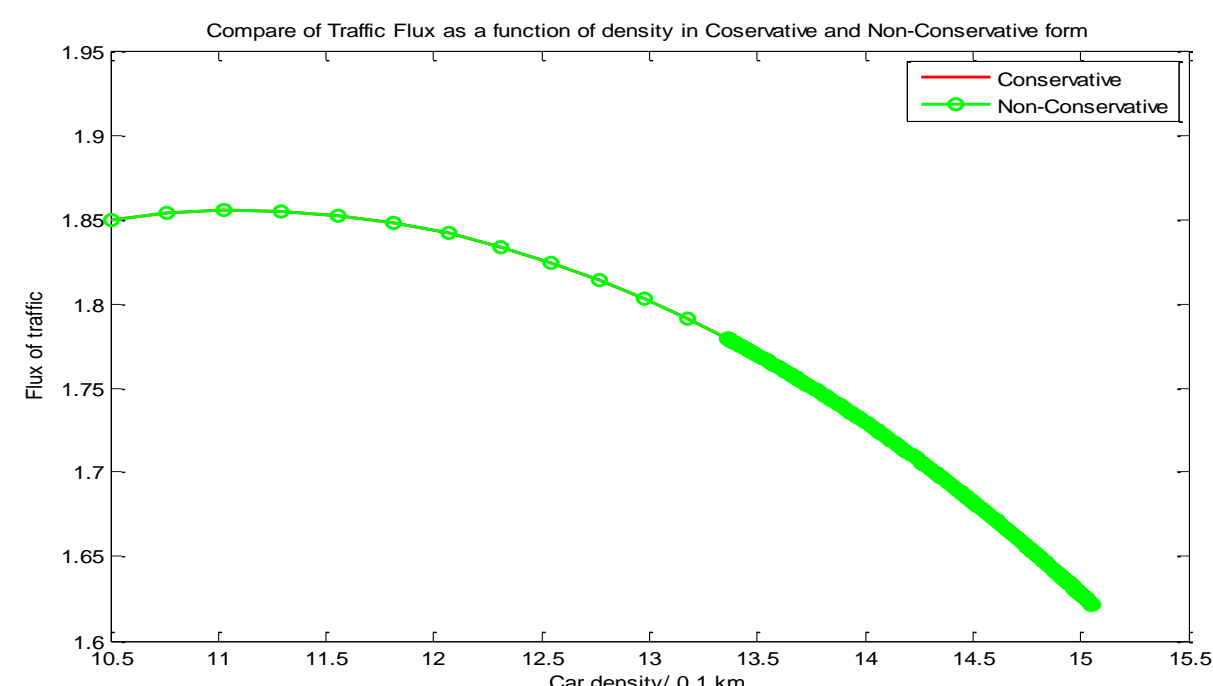

Fig-5(ii): Traffic Flux as a function of density (non-linear case) conservative and non-conservative form 
The computed flux is plotted with respect to the density profile by the flux-density relationship formula: linear case $q(\rho)=v_{\max }\left(\rho-\frac{\rho^{2}}{\rho_{\max }}\right)$ and non-linear case $q(\rho)=v_{\max }\left(\rho-\frac{\rho^{3}}{\rho_{\max }^{2}}\right)$, which is parabolic and concave function in the range $0 \leq \rho \leq \rho_{\max }$. Figure-5(i)

$$
\begin{gathered}
\rho(0, x)=\rho_{\mathrm{o}}(x)=15 \sin \left(\frac{x}{4}\right)+16 \text { we have } \\
c=v_{\max }\left(1-\frac{2\left(15 \sin \left(\frac{x}{4}\right)+16\right)}{\rho_{\max }}\right) \\
\Rightarrow \rho(t, x)=15 \sin \left(\frac{(x-c t)}{4}\right)+16
\end{gathered}
$$

We prescribe the corresponding boundary value for EUDS by the equation

$$
\rho_{a}(t)=\rho\left(t, x_{a}\right)=15 \sin \left(\frac{\left(x_{a}-c t\right)}{4}\right)+16
$$

We compute the relative error in $L_{1}$-norm defined by $\|e\|_{1}=\frac{\left\|\rho_{e}-\rho_{n}\right\|_{1}}{\left\|\rho_{e}\right\|_{1}}$ for all time $\rho_{e}$ is the exact solution and $\rho_{n}$ is the numerical solution computed by finite difference scheme. and 5(ii) present the graphs of flux with respect to the density in conservative and non-conservative form of a traffic flow model.

\subsection{Error Estimation of Numerical Scheme}

In order to perform error estimation, we consider exact solution (4) with initial condition i.e. non-linear function
Figure-6 shows the comparison of relative errors between explicit upwind difference scheme of conservative and non-conservative form. From figure we see that the relative error EUDS of conservative form, which remains 0.006 and the relative error nonconservative form remains 0.005 which is quite acceptable. So, non-conservative form provides more accurate results than conservative form. Figure-7 presents that the density $(\rho)$ error is decreasing with respect to the smaller discretization parameters $\Delta t$ and $\Delta x$ which shows the convergence of explicit upwind difference scheme of conservative and non-conservative form. We observe that as we increase number of grid points the error is decreasing and also shows the rate of convergence of the numerical solutions.

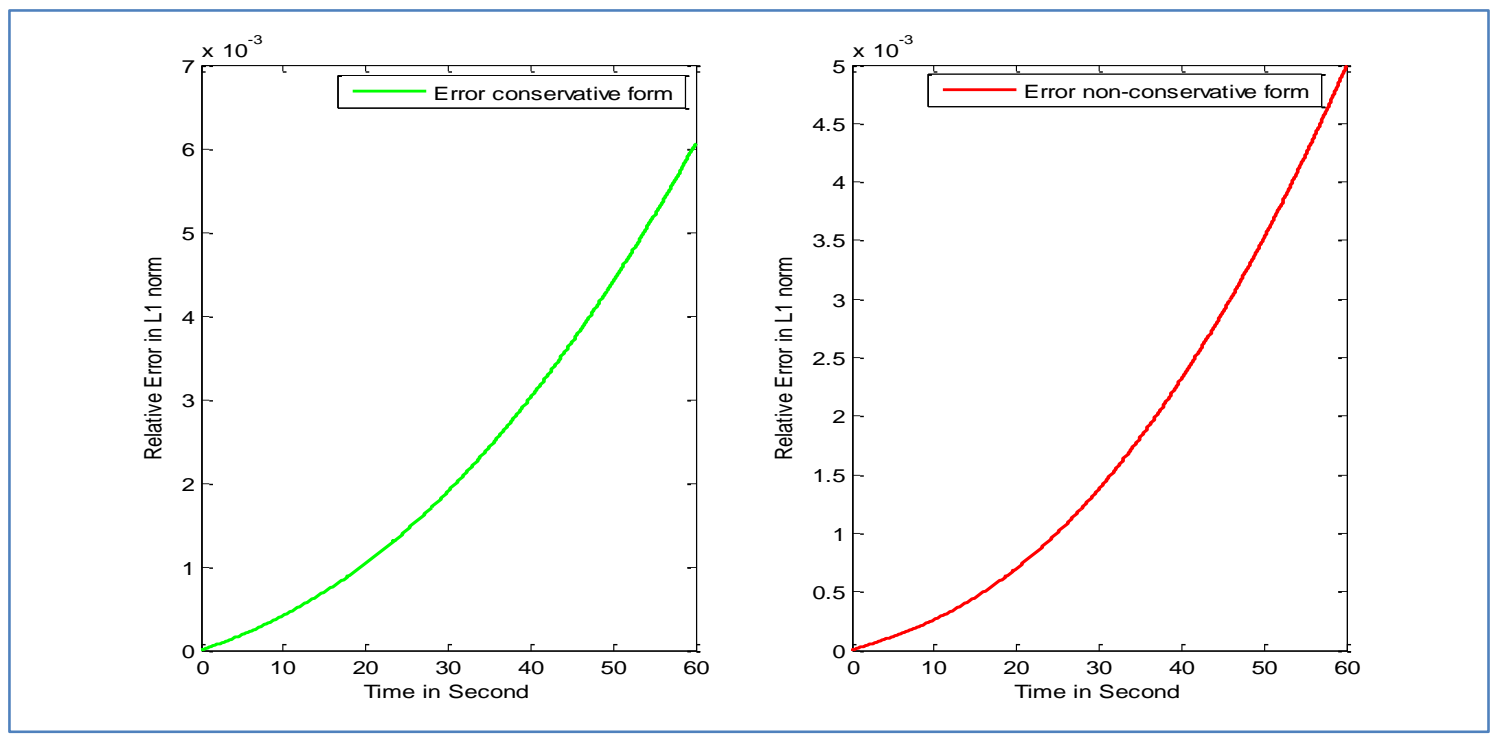

Fig-6: Comparison of relative errors between conservative and non-conservative form 


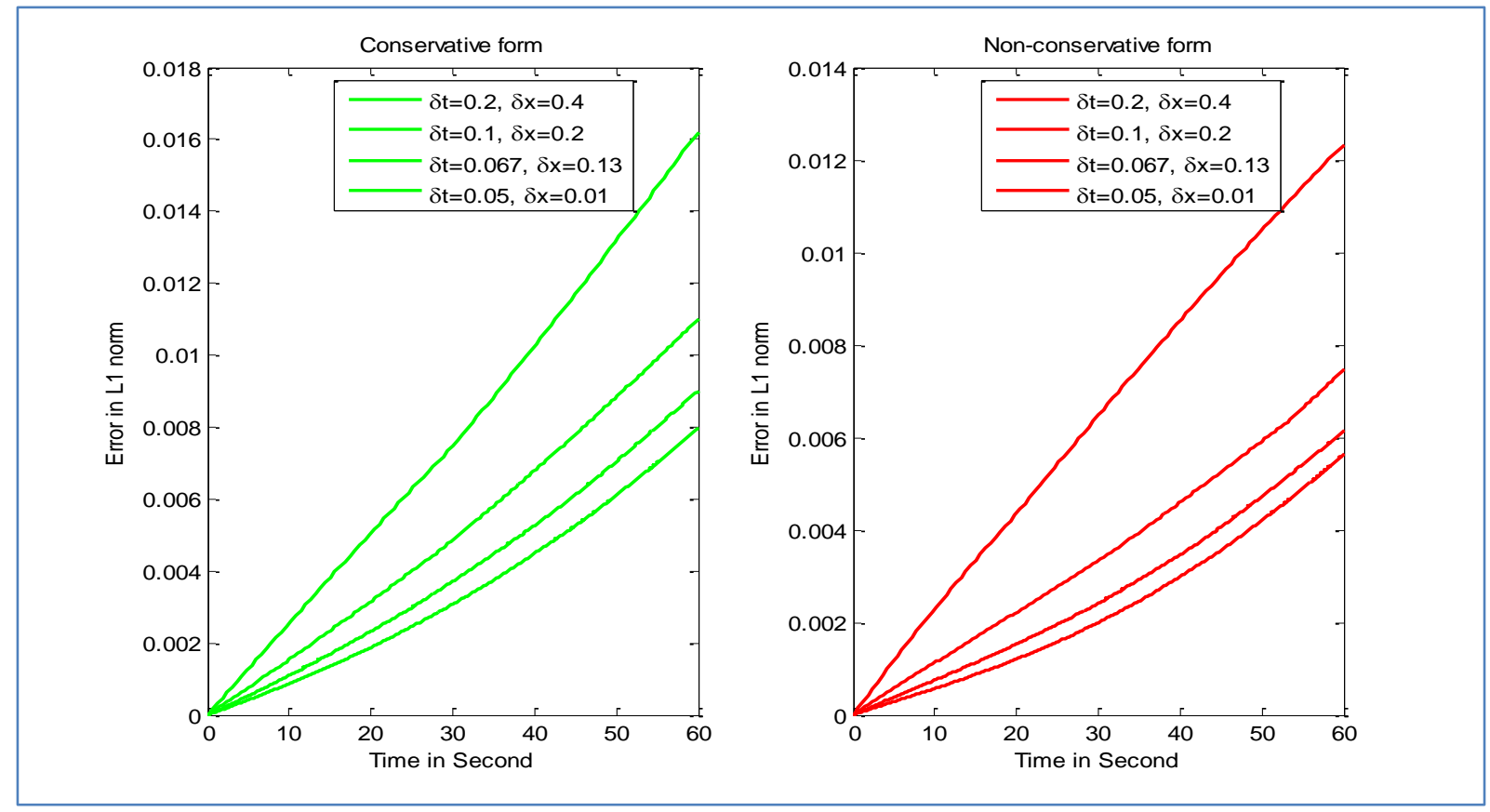

Fig-7: Comparison of convergence errors between conservative and non-conservative form

\section{CONCLUSION}

The finite difference scheme has been used to solve the traffic flow model. We have demonstrated numerical solution by using EUDS of conservative and non-conservative form. We establish stability conditions of EUDS. The numerical simulation results verified some qualitative traffic flow behavior for various traffic parameters. The outcome of different parameters has also been presented. Also we observe that the relative error EUDS of non-conservative form is much less than that of conservative form and the rate of convergence of EUDS non-conservative form is much higher than that of EUDS conservative form.

\section{REFERENCES}

1. Bretti, G., Natalini, R., \& Piccoli, B. (2007). "A Fluid-Dynamic Traffic Model on Road Networks", Comput Methods Eng., CIMNE, Barcelona, Spain. $14 ; 139-172$.

2. Andallah, L.S., Ali, S., Gani, M.O., Pandit, M.K. and Akhter, J. (2009). A Finite Difference Scheme for a Traffic Flow Model Based on a Linear Velocity-Density Function. Jahangirnagar University Journal of Science, 32, 61-71.

3. Kuhne, R., \& Michalopoulos, P. (1997). Continuum Flow Models.
4. Haberman, R. (1977). Mathematical Models. Prentice-Hall, Inc., Delhi.

5. Klar, A., Kuhne, R.D., \& Wegener, R. (1996). Mathematical Models for Vehicular Traffic. Technical University of Kaiserslautern, Dept. of Math., TU of Kaiserlautern, Germany.

6. Zhang, H. M. (2001). "A finite difference approximation of non-equilibrium traffic flow model", Transportation Research Part-B: Methodological, 35(4), (Elsevier), 337-365.

7. Dym, C. L. (2004). "Principles of Mathematical Modeling", Academic press.

8. Larsson, S., \& Thomée, V. (2003). Partial differential equations with numerical methods (Vol. 45, pp. x+-259). Berlin: Springer.

9. Leveque, R.J. (1992). Numerical Methods for Conservation Laws. 2nd Edition, Springer, Berlin.

10. Daganzo, C.F. (1995). A Finite Difference Approximation of the Kinematic Wave Model of Traffic Flow. Transportation Research Part B: Methodological, 29, 261-276.

11. Kabir, M. H., Gani, M. O., \& Andallah, L. S. (2010). Numerical simulation of a mathematical traffic flow model based on a nonlinear velocitydensity function. Journal of Bangladesh academy of sciences, 34(1), 15-22. 